\title{
Relativistic MOND from modified energetics
}

\author{
Durmuş Ali Demir ${ }^{\mathrm{a}}$, Canan Nurhan Karahan ${ }^{\mathrm{b}}$ \\ İzmir Institute of Technology, 35430 İzmir, Turkey
}

Received: 26 August 2014 / Accepted: 26 November 2014 / Published online: 13 December 2014

(C) The Author(s) 2014. This article is published with open access at Springerlink.com

\begin{abstract}
We begin to investigate the question of what modifications in the energy-momentum tensor can yield the correct MOND regime. As a starting study, we refrain from insisting on an action principle and focus exclusively on the equations of motion. The present work, despite the absence of an explicit action functional, can be regarded to extend Milgrom's modified inertia approach to relativistic domain. Our results show that a proper MOND limit arises if the energymomentum tensor is modified to involve the determinant of the metric tensor in reference to the flat metric, where the latter is dynamically generated as in the gravitational Higgs mechanism. This modified energy-momentum tensor is conserved in both Newtonian and MONDian regimes.
\end{abstract}

\section{Introduction}

Observations of several decades, ranging from the initial measurements by Oort (see the discussion in [1]) to the primal ones by Rubin [2,3], have shown that galaxies exhibit flat rotation curves, manifestly violating the Keplerian dynamics. This universal anomalous dynamics has been interpreted in two distinct ways. The first, first proposed by Zwicky [4] in 1933, refers to the dark matter (DM) hypothesis. According to the DM paradigm, there must be a distribution of non-shining matter at the outer skirts of galaxies to yield the approximately constant velocities after particular distances from the center of galaxies. The DM hypothesis provides viable explanations not only for flat rotation curves but also for various cosmological and astronomical observations describing different phases of the evolution of the Universe. Several experimental groups have been searching for DM particles by utilizing various detection methods (see the recent review volumes $[5,6])$. So far, no signal of DM has been observed.

\footnotetext{
a e-mail: demir@physics.iztech.edu.tr

b e-mail: cananduzturk@iyte.edu.tr
}

The second interpretation, first proposed by Milgrom [79] in 1983, postulates that the observed flat rotation curves result from modifications in the Newtonian laws of motion. In this approach, instead of adding unknown ingredients to the galactic matter, one exercises modifications in the equations of motion which dominate at the skirts of the galaxies. To this end, Newton's law of motion $\mathbf{F}=m \mathbf{a}$ changes to

$\mathbf{F}=m \mu\left(\frac{|\mathbf{a}|}{a_{0}}\right) \mathbf{a}$

where $\mathbf{F}$ is the net force acting on the material point which has inertia $m$ and acceleration $\mathbf{a}$ (with $\left.|\mathbf{a}|^{2}=\mathbf{a} \cdot \mathbf{a}\right)$. This dynamical equation, structuring Milgrom's MOND theory [7-9], is characterized by the empirical function $\mu\left(|\mathbf{a}| / a_{0}\right)$ where $a_{0} \simeq 1.2 \times 10^{-10} \mathrm{~ms}^{-2}$ is a constant acceleration scale for all galaxies [10]. It appears in (1) as a critical acceleration scale set galactically by the mass $M$ and radius $R$ of the galaxy as $\left(G_{N} M\right) / R^{2} \simeq a_{0}$ and cosmologically by the present-day value $H_{0}$ of the Hubble parameter as $\left(c H_{0}\right) / 2 \pi \simeq a_{0}[11]$.

The heart of the MOND theory is the empirical function $\mu\left(|\mathbf{a}| / a_{0}\right)$. There is yet no dynamical theory for it; however, its asymptotic behavior is not difficult to guess:

$\mu(x) \asymp \begin{cases}1 & \text { if } x>1, \\ x & \text { if } x<1,\end{cases}$

if all the successes of the Newtonian theory are to be maintained. Here $x$ does not need to be very large or small compared to unity because $\mu(x)$ can attain its asymptotics even when $x$ is close to unity. For instance, the empirical form

$\mu(x)=\frac{x\left(\frac{3}{2}\right)^{\frac{2}{n^{2}}}}{\left(\frac{1}{1+x^{n}}+x^{n}\right)^{\frac{1}{n}}}$

facilitates the asymptotics in (2) almost independently of $x$ provided that $n$ is large. Indeed, taking $n=50$ one finds $\mu(x)=0.700,0.900,0.986,0.996,1.000,1.000$ for $x=0.7$, $0.9,0.99,1.01,1.1,2.0$, respectively. 
The behavior in (2) ensures that matter in the galaxy exhibits flat rotation curves far away from the galactic center. Indeed, in the limit of small accelerations the equation of motion (1) takes the form

$\mathbf{F}=m \frac{|\mathbf{a}|}{a_{0}} \mathbf{a}$,

so that at large radii $R$ corresponding to the outer skirts of the galaxy one does not find the Keplerian law $|\mathbf{F}|=\left(m v^{2}\right) / R$ but $|\mathbf{F}|=\left(m v^{4}\right) /\left(a_{0} R^{2}\right)$, which yields the constant speed

$v^{4}=G_{N} M a_{0}$

for $|\mathbf{F}|=\left(G_{N} m M\right) / R^{2}$. This relation accounts for the observed flat rotation curves [2,3,12-14]. The constant speed (5) is the reason for and result from the whole idea of MOND. It depends crucially on the behavior of the empirical function (2) at low accelerations.

The empirical MOND relation in (1), supported by (2) and (3), needs be formulated at a more fundamental level. In this regard, there arise two different interpretations. In the first, after setting $\mathbf{a}=-\nabla \phi_{g}$ with $\phi_{g}$ being the gravitational potential, one formulates MOND as a modification in gravitational laws (see the reviews [15-17]). In this case, one is necessarily led to modified Newtonian gravity $[18,19]$ or General Relativity (GR) extended by geometrical scalar and vector fields [20-25]. Besides, there are alternative approaches based on $f(R)$ gravity [26-29], bimetric gravity $[30,31]$, time foliation $[32,33]$, nonlocal metric theories [34,35], Galileons [36], and Horava-Lifshitz gravity [37]. In general, modified gravity theories introduced to replace the DM necessarily lead to a MONDian structure.

In the second interpretation, one conceives of the equation of motion (1) as defining an acceleration-dependent inertia $m(|\mathbf{a}|)=m \mu\left(|\mathbf{a}| / a_{0}\right)$. This approach, the modified inertia approach proposed in $[38,39]$, in the non-relativistic limit, keeps gravitational laws unchanged yet lets in nonlinear kinetic terms. In this framework, it is found that the kinetic term of the point mass involves all derivatives of acceleration [38-41], yet it is stable and respects causality $[15-17,34,35]$. In the present work, we pursue this modified inertia viewpoint to generalize it to general-relativistic domain. The experience from non-relativistic study [38,39] ensures that forming an action functional must be difficult, if not impossible, in the relativistic domain. We thus focus exclusively on the equations of motion without specifying an action principle to derive them.

\section{Modified energetics}

As the beginning phase of a study program aiming at finding dynamical alternatives to modified gravity models of relativistic MOND [15-17], in this section we study grav- itational field equations where the MOND phase is understood as changes in the matter energy-momentum tensor. This approach, aiming at carrying Milgrom's modified inertia approach [38,39] into the relativistic domain at the level of equations of motion, is based on the matter energy-momentum tensor $T_{\mu \nu}^{(N)}$ in the Newtonian domain and exploits its expected non-conservation in the MOND regime to derive the MONDian dynamics in an empirical way. Having a complete knowledge of the interactions of matter, its energy-momentum tensor $T_{\mu \nu}^{(N)}$ (with energy density $T_{00}^{N}$, pressure $T_{i i}^{N}$, momentum density $T_{0 i}^{N}$ and shear stress $T_{i j}^{N}$ ) are strictly conserved in the Newtonian regime. However, the same $T_{\mu \nu}^{(N)}$ is not conserved in the MONDian regime because matter develops extra interactions even if one is not able to know them explicitly. Those extra interactions generalize $T_{\mu \nu}^{(N)}$ to a conserved energy-momentum tensor $T_{\mu \nu}$ which can be approached only empirically in the absence of a complete dynamical model (see $[43,44]$ for a similar approach to a modified gravity framework for MOND). We now give an empirical implementation of this dynamical picture starting with the Einstein field equations,

$G_{\mu \nu}=8 \pi G_{N} T_{\mu \nu}$,

in which $T_{\mu v}$ is the conserved energy-momentum tensor of matter at all acceleration scales ranging from $|\mathbf{a}|=0$ to $|\mathbf{a}|=\infty$. In general, $T_{\mu \nu}$ is conserved on the equations of motion, and these equations necessarily encode the novel interactions of matter responsible for the MOND. However, those new interactions are not known and our knowledge of $T_{\mu \nu}$ is incomplete; we are able to know it only when $|\mathbf{a}|>a_{0}$, for which it equals $T_{\mu \nu}^{(N)}$. Consequently, on an empirical basis we write for $T_{\mu \nu}$

$T_{\mu \nu}=\mu(\mathfrak{a})\left[T_{\mu \nu}^{(N)}-Q g_{\mu \nu}\right]+Q g_{\mu \nu}$

where $\mu(x)$ is the MOND function in (2), $Q$ is a scalar, and $\mathfrak{a}$ is yet another scalar which is to be judiciously constructed to have the empirical limit

$\mathfrak{a} \stackrel{v \ll c}{\longrightarrow} \mathfrak{a}_{N R}=\frac{|\mathbf{a}|}{a_{0}}$

at non-relativistic energies. This correspondence between the relativistic ( $\mathfrak{a})$ and non-relativistic $(|\mathbf{a}|)$ regimes is crucial for the empirical structure in (7) to give a consistent framework.

Physically, the grand energy-momentum tensor $T_{\mu \nu}$ must correctly reproduce the Newtonian and MONDian regimes. This is analyzed case by case in Table 1 as a function of the divergence of $T_{\mu \nu}^{(N)}$. As suggested by the table, the underlying dynamics can be revealed after a proper understanding of $T_{\mu \nu}$ and this requires $T_{\mu \nu}^{(N)}, \mathfrak{a}(T)$ and $Q(T)$ to be constructed in detail. We detail these physical variables in the three consecutive subsections that follow. 
Table 1 The acceleration dependence of the energy-momentum tensor $T_{\mu \nu}$ of matter. In general, $\mathfrak{a}=\mathfrak{a}\left(T^{(N)}\right)$ and $Q=Q\left(T^{(N)}\right)$ are functions of the energy-momentum tensor $T_{\mu \nu}^{(N)}$. These scalars take appropriate values for Newtonian $\left(T_{\mu \nu}^{(N)}\right.$ is conserved) and MONDian $\left(T_{\mu \nu}^{(N)}\right.$ is not conserved) regimes. Namely, matter develops novel interactions (such as the higher-derivative kinetic terms, determined in $[38,39]$ in the non-relativistic regime) at small accelerations and its known energymomentum tensor $T_{\mu \nu}^{(N)}$ starts exhibiting non-conservation properties

\begin{tabular}{|c|c|c|c|}
\hline Acceleration & MOND function & Energy-momentum tensor & Matter dynamics \\
\hline $\mathfrak{a} \gtrsim 1$ & $\mu(\mathfrak{a}) \simeq 1$ & $\begin{array}{l}T_{\mu \nu} \simeq T_{\mu \nu}^{(N)} \\
\left(\nabla^{\mu} T_{\mu \nu}=0 \text { hence } \nabla^{\mu} T_{\mu \nu}^{(N)}=0\right)\end{array}$ & $\begin{array}{l}\text { This is the 'Newtonian regime'. The acceleration } \\
\text { of matter is above } a_{0} \text { and } \mu(\mathfrak{a}) \text { ensures } \\
T_{\mu \nu} \simeq T_{\mu \nu}^{(N)} \text { so that } T_{\mu \nu}^{(N)} \text { is symmetric and } \\
\text { divergence-free }\left(\nabla^{\mu} T_{\mu \nu}^{(N)}=0\right) \text { in agreement } \\
\text { with (6). In the Newtonian regime thus } T_{\mu \nu}^{(N)} \\
\text { qualifies as the well-known conserved } \\
\text { energy-momentum tensor of matter }\end{array}$ \\
\hline $\mathfrak{a} \lesssim 1$ & $\mu(\mathfrak{a}) \simeq \mathfrak{a}$ & $\begin{array}{l}T_{\mu \nu} \not T_{\mu \nu}^{(N)} \\
\left(\nabla^{\mu} T_{\mu \nu}=0 \text { yet } \nabla^{\mu} T_{\mu \nu}^{(N)} \neq 0\right)\end{array}$ & $\begin{array}{l}\text { This is the 'MONDian regime'. The acceleration } \\
\text { of matter is below } a_{0} \text { and } \mu(\mathfrak{a}) \text { leads to } \\
T_{\mu \nu} \nsucceq T_{\mu \nu}^{(N)} \text { so that } T_{\mu \nu}^{(N)} \text { is symmetric yet not } \\
\text { divergence-free }\left(\nabla^{\mu} T_{\mu \nu}^{(N)} \neq 0\right) \text {. In the MOND } \\
\text { regime thus it is } T_{\mu \nu} \text { not } T_{\mu \nu}^{(N)} \text {, which qualifies } \\
\text { as the conserved energy-momentum tensor of } \\
\text { matter. In this small acceleration regime, matter } \\
\text { develops novel interactions that make } \\
\nabla^{\mu} T_{\mu \nu}^{(N)} \neq 0 \text { yet the scalars } \mathfrak{a} \text { and } Q \text { help } T_{\mu \nu} \\
\text { be conserved and give the observed flat rotation } \\
\text { curves }\end{array}$ \\
\hline
\end{tabular}

\subsection{Physical properties of $T_{\mu \nu}^{(N)}$}

It has been emphasized previously, specifically in Table 1, that $T_{\mu \nu}^{(N)}$ has the same form as the energy-momentum tensor of matter in the Newtonian regime yet it does not qualify as a true energy-momentum tensor in the MOND regime simply because its conservation is spoiled by novel interactions of matter that arise at accelerations below $a_{0}$. The higher-derivative self interactions studied in [38-41] form a concrete example of such effects. Let us consider, as an illustrative example, dust (pressureless matter having only energy density in the comoving frame) for which

$T_{\mu \nu}^{(N)}=\rho u_{\mu} u_{\nu}$

where $\rho$ and $u_{\mu}$ are the energy density and velocity, respectively. (One recalls that $T_{\mu \nu}^{(N)}=\int d \tau \rho u_{\mu} u_{v}$ for a relativistic particle with trajectory $y_{\mu}(\tau)$ and energy density $\left.\rho=m c^{2} \delta^{4}(x-y(\tau))\right)$. It is divergence-free, $\nabla^{\mu} T_{\mu \nu}^{(N)}=0$, because densities and flows of dust are all conserved. However, this conservation property holds only in normal circumstances where the Newtonian laws of motion are valid. In the MONDian regime, where dust develops higher-derivative kinetic interactions for instance, conservation breaks down, $\nabla^{\mu} T_{\mu \nu}^{(N)} \neq 0$. On dimensional grounds, it is likely to have a structure of the form

$\nabla^{\mu} T_{\mu \nu}^{(N)} \sim \rho a_{0} u_{\nu}$ in addition to terms involving derivatives of the acceleration. In the absence of an invariant action (like the non-relativistic model in $[38,39])$, this non-conservation can be understood neither in origin nor in structure $\left(\rho a_{0} u_{v}\right.$ in (10) is just an example). Therefore, our goal is not to construct a model of the non-conservation of $T_{\mu \nu}^{(N)}$, but to determine its consequences for structures and dynamics of $\mathfrak{a}$ and $Q$.

\subsection{Physical Properties of the Acceleration Scalar $\mathfrak{a}$}

The acceleration scalar $\mathfrak{a}$, which must have the nonrelativistic limit $\mathfrak{a}_{N R}$ given in (8), must be constructed judiciously to correctly cover the Newtonian and MONDian regimes. Hence, besides the crucial relation (8), it must have the following properties.

1. By our construction shown in Table $1, \mathfrak{a}$ must vary with the divergence of $T_{\mu \nu}^{(N)}$ as

$$
\begin{array}{lll}
\mathfrak{a}>1 & \text { if } & \nabla^{\mu} T_{\mu \nu}^{(N)}=0, \\
\mathfrak{a}<1 & \text { if } & \nabla^{\mu} T_{\mu \nu}^{(N)} \neq 0,
\end{array}
$$

while $\nabla^{\mu} T_{\mu \nu}=0$ in both cases.

2. Being a scalar field, $\mathfrak{a}$ involves contractions of the divergences of $T_{\mu \nu}^{(N)}$. This necessarily brings in the gravitational acceleration $\nabla \phi_{g}$ through the gravitational potential $\phi_{g}=-1-g_{00}$ arising in the Newtonian limit of the 
metric tensor $g_{\mu \nu}$. However, the presence of $\nabla \phi_{g}$ must be prohibited for $\mathfrak{a}$ to yield the kinetic acceleration in (8). It is easy to see that this cannot be accomplished without using an independent source of $\phi_{g}$ and the most natural source as such is the determinant $g=\operatorname{Det}\left(g_{\mu \nu}\right)$ of the metric tensor. However, being a scalar density rather than a scalar, $g$ cannot appear in $\mathfrak{a}$ by itself; it must be divided by another scalar density to achieve covariance. This other scalar density necessitates a new metric $\bar{g}_{\mu \nu}$, and naturally leads to a bimetrical picture (whose relevance for MOND has been discussed in [30,31]). Then the acceleration scalar possess the functional form

$\mathfrak{a}=\mathfrak{a}\left(a_{0}, \nabla^{\mu} T_{\mu \nu}^{(N)}, T_{\mu \nu}^{(N)}, g_{\mu \nu}, g_{\mu \nu} \bar{g}^{\mu \nu}, g / \bar{g}\right)$

where $\bar{g}=\operatorname{Det}\left(\bar{g}_{\mu \nu}\right)$ arises as an additional variable to be dynamically determined.

These two points plus (8) must be taken into account in formulating $\mathfrak{a}$. However, the formulation process becomes utterly incomplete unless the additional metric $\bar{g}_{\mu \nu}$ is demystified. In the two subsections that follow, we first study $\bar{g}_{\mu \nu}$ and then construct a model of $\mathfrak{a}$.

\subsubsection{Construction of $\bar{g}_{\mu \nu}$}

The second metric tensor $\bar{g}_{\mu \nu}$, required to eliminate the gravitational acceleration $\nabla \phi_{g}$ from the acceleration scalar $\mathfrak{a}$, can be ascribed different structures depending on the underlying dynamics. For instance, one may consider identifying it with $T_{\mu \nu}^{(N)}$ itself but this attempt fails because its determinant vanishes in the case of dust (see Eq. 9 above). Alternatively, one may take $\bar{g}_{\mu \nu}$ as a second metric tensor with its own curvature and dynamics, but this setup, as was already elaborated by Milgrom in [30,31] (see also [42]), gives a modified gravity theory for MOND. This and other possible modified gravity models fall outside the scope of the present work because the goal here is to develop a dynamical approach to relativistic MOND similar in philosophy to Milgrom's modified inertia approach $[38,39]$.

Our approach to $\bar{g}_{\mu \nu}$ is dynamical rather than geometrical. In other words, the dynamics underlying the asymptotics in Table 1 and structures in (12) proceed not only with $T_{\mu \nu}^{(N)}$ but also $\bar{g}_{\mu \nu}$. Thus, $\bar{g}_{\mu \nu}$ is a low-acceleration dynamical field, maybe one of many as such, which facilitates the MOND regime. In modeling the dynamics, we interpret the coupling $g^{\mu \nu} \bar{g}_{\mu \nu}$ between the two metrics as the kinetic term of four real scalars $\phi^{m}(m=0, \ldots, 3)$, and we construct the defining relation

$\bar{g}_{\mu \nu}=\frac{1}{M^{4}} \eta_{m n} \partial_{\mu} \phi^{m} \partial_{\nu} \phi^{n}$ where $\eta_{m n}$ is the flat Minkowski metric, and hence the scalar spectrum contains a ghosty (negative kinetic term) mode. We assume that $\phi^{m}$ develops the nontrivial backgrounds

$\left\langle\bar{g}_{\mu \nu}\right\rangle= \begin{cases}0 & \text { if }\left\langle\phi^{m}\right\rangle=0, \\ \eta_{\mu \nu} & \text { if }\left\langle\phi^{m}\right\rangle=M^{2} x^{m},\end{cases}$

depending on whether the diffeomorphism invariance is exact $\left(\left\langle\phi^{m}\right\rangle=0\right)$ or spontaneously broken $\left(\left\langle\phi^{m}\right\rangle=M^{2} x^{a}\right)$ in the vacuum state governed by the vacuum expectation value $\left\langle\phi^{a}\right\rangle$ of the scalars. Here, the scale $M$ is around $a_{0}$. The dynamics leading to (14) can be known only in a setting where all interactions of matter and extra fields like $\phi^{a}$ are specified. The diffeomorphism-breaking vacuum here sets the flat Minkowski metric $\eta_{\mu \nu}$ as the background metric about which $g_{\mu \nu}$ can be expanded in a perturbation series.

This induction mechanism is similar to what happens in the gravitational Higgs mechanism [46-50], in which a second metric tensor $\bar{g}_{\mu \nu}$ is needed for writing a sensible graviton mass term through the kinetic term $g^{\mu \nu} \bar{g}_{\mu \nu}$ of scalars and through the ratio of the determinants $g / \bar{g}$. Nevertheless, as was thoroughly analyzed in [50], these two contributions, instead of adding, can cancel each other to keep the graviton massless, or equivalently, gravity unmodified. This does not mean that the metric tensors in (14) do not participate in other physical processes. Indeed, they can well generate our targeted structures involving the gravitational acceleration $\nabla \phi$. Consequently, we associate the metric tensors in (14) with the two phases of motion as

$\left\langle\bar{g}_{\mu \nu}\right\rangle=0 \Longrightarrow$ Newtonian regime,

$\left\langle\bar{g}_{\mu \nu}\right\rangle=\eta_{\mu \nu} \quad \Longrightarrow \quad$ MONDian regime,

keeping in mind that gravity is not necessarily massive. Indeed, the model of [50] offers a wide parameter space to set $V_{1}^{\prime}(4)=0$ in Eq. (26) and $\zeta V_{1}^{\prime}(4)=0$ in Eq. (27). Moreover, potential terms in Eq. (11) give enough freedom to realize massless and massive gravity phases. Therefore, as will be proven below, the MOND regime can be realized by using the metrics in (14) without the necessity of modifying gravity.

\subsubsection{Construction of $\mathfrak{a}$}

Having fixed all the variables in (12), we now start formulating the acceleration scalar $\mathfrak{a}$. The kinetic term $g^{\mu \nu} \bar{g}_{\mu \nu}$ of the scalars does not contribute to $\nabla \phi_{g}$, and hence, the argument of $\mathfrak{a}$ in (12) represents the optimal list of dynamical variables. Out of various possibilities, we consider for $\mathfrak{a}$ a simple structure,

$$
\begin{aligned}
\mathfrak{a}^{2} a_{0}^{2}\left(T^{(N)}\right)^{2}= & \nabla^{\alpha} T_{\alpha}^{(N)}{ }_{\alpha}^{\beta} \nabla_{\theta \beta}^{(N)} \\
& +c_{1}\left(T^{(N)}\right)^{2} \nabla_{\alpha}\left(\frac{g}{\bar{g}}\right) \nabla^{\alpha}\left(\frac{g}{\bar{g}}\right) \\
& +c_{2} T^{(N)} \nabla^{\alpha}\left(\frac{g}{\bar{g}}\right) \nabla^{\theta} T_{\theta \alpha}^{(N)}
\end{aligned}
$$


where all indices are raised and lowered with $g_{\alpha \beta}$ so that $T^{(N)}=g^{\alpha \beta} T_{\alpha \beta}^{(N)}$ is the trace of the matter energy-momentum tensor in the Newtonian domain. Here, the dimensionless constants $c_{1,2}$ will be fixed in the weak field limit by imposing (8). The presence of the metric determinants in (16) is crucially important for MOND because gravitational acceleration $\nabla \phi_{g}$ is generated by the derivatives of $g / \bar{g}$ (not $g^{\mu \nu} \bar{g}_{\mu \nu}$, for instance).

Having fixed its functional form in (16), we now start checking if $\mathfrak{a}$ satisfies its defining asymptotics in (2) and Table 1. This requires its evaluation in the two vacua in (14) since they correspond to the Newtonian and MONDian regimes as indicated in (15).

1. $\left\langle\bar{g}_{\mu \nu}\right\rangle=0$ and $\nabla^{\mu} T_{\mu \nu}^{(N)}=0$. In this vacuum, $\langle\bar{g}\rangle$ vanishes identically and, as follows from (16), $\mathfrak{a}$ becomes infinitely large thanks to the fact that $c_{1,2}>0$, as will be proven below. Now, having found $\mathfrak{a}>1$, one gets $\mu(\mathfrak{a}) \simeq 1$ and this gives $T_{\mu \nu} \simeq T_{\mu \nu}^{(N)}$ from (7). Thus, the Einstein field equations (6) reduce to

$$
G_{\mu \nu}=8 \pi G_{N} T_{\mu \nu}^{(N)}
$$

in which consistency of the Bianchi identity on $G_{\mu \nu}$ is maintained by the conservation of $T_{\mu \nu}^{(N)}$. This conservation, $\nabla^{\mu} T_{\mu \nu}^{(N)}=0$, gives the usual Newtonian equations for free fall,

$\mathbf{a}=-\nabla \phi_{g}$

for a dust distribution characterized by the energymomentum tensor in (9). Clearly, this equation holds if the metric tensor takes the form

$g_{\mu \nu}=$ Diag. $\left(-\left(1+2 \phi_{g}\right), 1,1,1\right)_{\mu \nu}$,

as appropriate for the non-relativistic limit.

In conclusion, as conjectured in Eq. (15), the minimum energy configuration $\langle\bar{g}\rangle$ gives rise to the Newtonian regime for motion. Small perturbations about this vacuum make $\bar{g} \neq 0$ but this determinant is expected to be sufficiently small to secure the Newtonian regime, $\mathfrak{a}>1$.

2. $\left\langle\bar{g}_{\mu \nu}\right\rangle=\eta_{\mu \nu}$ and $\nabla^{\mu} T_{\mu \nu}^{(N)} \neq 0$. In this vacuum, in the non-relativistic limit in which the metric tensor is given by (19), the acceleration scalar defined in (16) becomes

$$
\begin{aligned}
\mathfrak{a}_{N R}^{2}= & \frac{\mathbf{a} \cdot \mathbf{a}}{a_{0}^{2}}+\left(2-c_{2}\right) \frac{\mathbf{a} \cdot \nabla \phi_{g}}{a_{0}^{2}} \\
& +\left(1-c_{2}+c_{1}\right) \frac{\nabla \phi_{g} \cdot \nabla \phi_{g}}{a_{0}^{2}}
\end{aligned}
$$

for dust whose energy-momentum tensor is given partly by (9) and partly by extra interactions occurring in the low-acceleration regime. It is due to this alleged extra piece that $T_{\mu \nu}^{(N)}$ in (9) satisfies $\nabla^{\mu} T_{\mu \nu}^{(N)} \neq 0$.

It is clear that the acceleration scalar exhibits correct nonrelativistic limit if

$c_{1}=1, \quad c_{2}=2$,

because then the last two terms of (20) drop out to enable the required limit in (8). Thus, the construction in (16) for $\mathfrak{a}$ does indeed reduce to the acceleration of the point mass rather than the gravitational acceleration $-\nabla \phi_{g}$. The non-relativistic result in (20), which holds for $\mathfrak{a}<1$ or equivalently $|\mathbf{a}|<a_{0}$, entails $\mu(\mathfrak{a}) \simeq \mathfrak{a}$, so that the Einstein field equations (6) take the form

$G_{\mu \nu}=8 \pi G_{N}\left\{\mathfrak{a}\left[T_{N \mu \nu}-Q g_{\mu \nu}\right]+Q g_{\mu \nu}\right\}$

where the scalar field $Q$ is to be chosen judiciously to make the right-hand side have a vanishing divergence. This constraint, ensuring conservation of $T_{\mu \nu}$, can be difficult to satisfy if $Q$ does not involve $T_{\mu \nu}^{(N}$ and $g / \bar{g}$. As a plausible structure, we set

$Q=\frac{g T^{(N)}}{\bar{g}}$

where one can of course consider alternative structures giving similar results in the non-relativistic limit. In $\left\langle\bar{g}_{\mu \nu}\right\rangle=\eta_{\mu \nu}$ vacuum, in the non-relativistic limit, conservation of $T_{\mu \nu}$ gives

$$
\begin{aligned}
\nabla_{\mu} & \left(\frac{|\mathbf{a}|}{a_{0}}\right)\left\{\rho u^{\mu} u^{j}-\rho\left(\frac{g}{\bar{g}}\right) g^{\mu j}\right\} \\
= & -\frac{|\mathbf{a}|}{a_{0}}\left\{\rho\left(\mathbf{a}^{j}+\nabla^{j} \phi_{g}\right)-\rho \nabla^{j} \phi_{g}-\left(1+2 \phi_{g}\right) \nabla_{\mu} \rho g^{\mu j}\right\} \\
& -\rho \nabla^{j} \phi_{g}-\left(1+2 \phi_{g}\right) \nabla_{\mu} \rho g^{\mu j}
\end{aligned}
$$

where the metric tensor is given by (19). This differential equation is too involved to suggest the MOND dynamics. Nevertheless, a closer look reveals that, if (i) the energy density $\rho$ varies slowly in space $\left(|\nabla \rho| \ll \rho\left|\nabla \phi_{g}\right|\right)$ and if (ii) acceleration a varies slowly both in space and time $\left(\left|\nabla_{\mu} \rho\right| \ll \rho\left|\nabla_{\mu} \phi_{g}\right|\right)$, then one gets from (24)

$$
\frac{|\mathbf{a}|}{a_{0}} \mathbf{a}=-\nabla \phi_{g},
$$

which is the desired MOND relation given in Eq. (4).

3. Non-conservation of $T_{N}^{\mu \nu}$. Having obtained motion equations in the two regimes of $\bar{g}_{\mu \nu}$, we now turn to a discussion of the non-conservation of $T_{N}^{\mu \nu}$. In view of the dis- 
cussions summarized in Table 1, the energy-momentum tensor $T_{\mu \nu}$, introduced in (6) and defined in (7), is always conserved. This is necessary for the consistency of the gravitational field equations (6). The $T_{N}^{\mu \nu}$ tensor, however, is conserved only in the Newtonian regime. To see how these conservation features hold, it proves useful to examine the divergence of $T_{N}^{\mu \nu}$,

$\nabla_{\mu} T_{N}^{\mu \nu}=f_{N}^{v}$

where

$$
\begin{aligned}
f_{N}^{v}= & -\left[\nabla_{\alpha} \ln \mu(\mathfrak{a})\right]\left(T_{N}^{\alpha \nu}-Q g^{\alpha \nu}\right) \\
& +\left(1-\frac{1}{\mu(\mathfrak{a})}\right) \nabla^{v} Q,
\end{aligned}
$$

as follows from (6) with (7). It is obvious that, in the Newtonian regime, $\mu(\mathfrak{a}) \rightarrow 1$ and $f_{N}^{v}$ vanishes identically to ensure conservation of $T_{N}^{\mu \nu}$. In the MONDian regime, however, $\mu(\mathfrak{a}) \rightarrow \mathfrak{a} \neq 1$, and $f_{N}^{v}$ stays non-vanishing. This prohibits conservation of $T_{N}^{\mu \nu}$. These features are precisely the ones listed in Table 1 . The MONDian force is consistent with (22). Since $\mathfrak{a}$ is related to $\bar{g}_{\mu \nu}$ as in (16), the second metric $\bar{g}_{\mu \nu}$ turns out to be a fundamental ingredient of the entire formalism. Not surprisingly, effective forces similar to $f_{N}^{v}$ also arise in modified gravity theories which couple curvature and energy-momentum tensor $T_{N}^{\mu v}$ directly [51-53].

In this section, we have succeeded to get the MONDian dynamics starting from (6) by defining the acceleration scalar $\mathfrak{a}$ as in (16), the $Q$ scalar as in (23), and the second metric tensor as in (13). Moreover, we have explicitly ensured conservation of the total energy-momentum tensor $T^{\mu \nu}$, while determining effective MOND force associated with the nonconservation of $T_{N}^{\mu \nu}$. The analysis here provides an existence proof.

\section{Conclusion and future prospects}

In the present paper, we reported our results on relativistic MOND as derived from modified dynamics rather than modified gravity. Our approach is an empirical one and gives the beginning stage of a general investigation of relativistic MOND. The formalism developed, though lacking an action principle, can be regarded as generalizing Milgrom's modified inertia approach [38-41] to the relativistic domain. It is based on the energy-momentum tensor of matter. The reason for this is that the energy-momentum tensor of matter in the Newtonian regime, which necessarily loses its conservation property due to extra interactions occurring at sub-
Hubble accelerations, seems to provide the correct path way to quadratic acceleration in the MOND regime. In fact, this dynamical structure cannot follow from other sources such as potentials, metric tensor, and curvature tensor. The main observation behind our approach is that matter possesses its usual energy-momentum tensor under the usual circumstances where the Newtonian laws hold. However, the same matter, at exceedingly small accelerations below the Hubble scale, develops novel interactions causing non-conservation of its energy-momentum tensor, and it is with these interactions that MONDian dynamics arises. Our empirical relativistic model is essentially a bimetric theory. However, our approach to the second metric tensor mimics models of the gravitational Higgs mechanism in which the vacuum expectation value of the second metric tensor equals the flat Minkowski metric, and it provides the requisite terms clearing the gravitational acceleration contributions to enable the quadratic acceleration piece needed for MOND.

The present study can be extended in various respects for rectifying and improving the present model.

- In the present work we have taken matter at the skirts of galaxies as dust. For an accurate analysis of the matter distribution, however, one may need to extend it to a perfect fluid and other forms of matter.

- In obtaining the MOND equation of motion (25) we have neglected contributions from the spatial variation of $\rho$. The situation can be improved by incorporating such terms from (24). The effect can be pronounced especially at the arms of spirals where the dust density changes sharply.

These points are currently under investigation in [54].

Last but not least, the present model would be grossly improved if an invariant action could be written. The alleged action, which must directly generalize Milgrom's modified inertia approach in $[38,39]$ to relativistic velocities could be too complicated to construct due mainly to the presence of the fixed acceleration scale $a_{0}$. It might necessitate $a_{0}$ to be included in relativistic transformations.

Acknowledgments We are grateful to Ismail Turan for fruitful discussions

Open Access This article is distributed under the terms of the Creative Commons Attribution License which permits any use, distribution, and reproduction in any medium, provided the original author(s) and the source are credited.

Funded by $\mathrm{SCOAP}^{3}$ / License Version CC BY 4.0.

\section{References}

1. K. Kuijken, G. Gilmore, Mon. Not. R. Astron. Soc. 239, 605 (1989)

2. V.C. Rubin, W.K. Ford Jr, Astrophys. J. 159, 379 (1970) 
3. V.C. Rubin, N. Thonnard, W.K. Ford Jr, Astrophys. J. 238, 471 (1980)

4. F. Zwicky, Helvetica Physica Acta 6, 110 âĂŞ127 (1933)

5. G. Bertone et al., Particle Dark Matter: Observations. Models and Searches (Cambridge Univ. Pr, Cambridge, 2010), p. 738

6. N. Fornengo, Adv. Space Res. 41, 2010 (2008). astro-ph/0612786

7. M. Milgrom, ApJ 270, 365 (1983)

8. M. Milgrom, ApJ 270, 371 (1983)

9. M. Milgrom, ApJ 270, 384 (1983)

10. J. Ginse, Chaos Solitons Fract. 41, 1651-1660 (2009)

11. D.A. Easson, P.H. Frampton, G.F. Smoot, Phys. Lett. B 696(3), 273-277 (2011)

12. R.H. Sanders, M.A.W. Verheijen, ApJ 503, 97-108 (1998)

13. R.H. Sanders, E. Noordermeer, MNRAS 379, 702-710 (2007)

14. J.R. Brownstein, J.W. Moffat, ApJ 636, 721-741 (2006)

15. J.-P. Bruneton, G. Esposito-Farese, Phys. Rev. D 76, 124012 (2007). arXiv:0705.4043 [gr-qc]. [Erratum-ibid. D 76, 129902 (2007)]

16. C. Skordis, Class. Quant. Grav. 26, 143001 (2009). arXiv:0903.3602 [astro-ph.CO]

17. B. Famaey, S. McGaugh, Living Rev. Rel. 15, 10 (2012). arXiv:1112.3960 [astro-ph.CO]

18. J. Bekenstein, M. Milgrom, Astrophys. J. 286, 7 (1984)

19. M. Milgrom, Mon. Not. Roy. Astron. Soc. 403, 886 (2010). arXiv:0911.5464 [astro-ph.CO]

20. R.H. Sanders, Astrophys. J. 480, 492 (1997). astro-ph/9612099

21. J. D. Bekenstein, Phys. Rev. D 70, 083509 (2004). [Erratum-ibid. D 71, 069901 (2005)] [astro-ph/0403694]

22. R.H. Sanders, Mon. Not. R. Astron. Soc. 363, 459 (2005). [astro-ph/0502222]

23. T.G. Zlosnik, P.G. Ferreira, G.D. Starkman, Phys. Rev. D 74, 044037 (2006). gr-qc/0606039

24. T.G. Zlosnik, P.G. Ferreira, G.D. Starkman, Phys. Rev. D 75, 044017 (2007). astro-ph/0607411

25. C.N. Karahan, A. Altas, D.A. Demir, Gen. Rel. Grav. 45, 319 (2013). arXiv: 1110.5168 [gr-qc]

26. O. Bertolami, C.G. Boehmer, T. Harko, F.S.N. Lobo, Phys. Rev. D 75, 104016 (2007). arXiv:0704.1733 [gr-qc]

27. O. Bertolami, J. Paramos, T. Harko, F.S.N. Lobo, arXiv:0811.2876 [gr-qc]

28. A. Stabile, G. Scelza, Phys. Rev. D 84, 124023 (2011). arXiv:1107.5351 [gr-qc]

29. T. Bernal, S. Capozziello, J.C. Hidalgo, S. Mendoza, Eur. Phys. J. C 71, 1794 (2011). arXiv:1108.5588 [astro-ph.CO]
30. M. Milgrom, Phys. Rev. D 80, 123536 (2009). arXiv:0912.0790 [gr-qc]

31. M. Milgrom, Mon. Not. Roy. Astron. Soc. 405, 1129 (2010). [arXiv:1001.4444 [astro-ph.CO]]

32. L. Blanchet, A.L. Tiec, arXiv:0807.1200 [astro-ph]

33. L. Blanchet, S. Marsat, Phys. Rev. D 84, 044056 (2011). arXiv: 1107.5264 [gr-qc]

34. M.E. Soussa, R.P. Woodard, Class. Quant. Grav. 20, 2737 (2003). astro-ph/0302030

35. C. Deffayet, G. Esposito-Farese, R.P. Woodard, Phys. Rev. D 84, 124054 (2011). arXiv:1106.4984 [gr-qc]

36. E. Babichev, C. Deffayet, G. Esposito-Farese, Phys. Rev. D 84, 061502 (2011). arXiv: 1106.2538 [gr-qc]

37. J.M. Romero, R. Bernal-Jaquez, O. Gonzalez-Gaxiola, Mod. Phys. Lett. A 25, 2501 (2010). arXiv:1003.0684 [hep-th]

38. M. Milgrom, Annals Phys. 229, 384 (1994). astro-ph/9303012

39. M. Milgrom, Phys. Lett. A 253, 273 (1999). astro-ph/9805346

40. J.M. Romero, A. Zamora, Phys. Rev. D 73, 027301 (2006). astro-ph/0601247

41. M.E. McCulloch, Mon. Not. R. Astron. Soc. 376, 338 (2007). astro-ph/0612599

42. M.E. Soussa, R.P. Woodard, Phys. Lett. B 578, 253 (2004). astro-ph/0307358

43. P. Mishra, T.P. Singh, Int. J. Mod. Phys. 21, 1242002 (2012). arXiv: 1205.3088 [gr-qc]

44. P. Mishra, T.P. Singh, Int. J. Mod. Phys. 21, 1242002 (2012) arXiv:1206.3322 [astro-ph.GA]

45. M. Forger, H. Romer, Ann. Phys. 309, 306 (2004). hep-th/0307199

46. R. Percacci, Nucl. Phys. B 353, 271 (1991). arXiv:0712.3545 [hepth]

47. G.'t Hooft, arXiv:0708.3184 [hep-th]

48. Z. Kakushadze, Int. J. Mod. Phys. A 23, 1581 (2008). arXiv:0709.1673 [hep-th]

49. Z. Kakushadze, Phys. Rev. D 77, 024001 (2008). arXiv:0710.1061 [hep-th]

50. D.A. Demir, N.K. Pak, Class. Quant. Grav. 26, 105018 (2009). arXiv:0904.0089 [hep-th]

51. Z. Haghani, T. Harko, F.S.N. Lobo, H.R. Sepangi, S. Shahidi, Phys. Rev. D 88(4), 044023 (2013). arXiv:1304.5957 [gr-qc]

52. M. Sharif, M. Zubair, JHEP 1312, 079 (2013). arXiv: 1306.3450 [gr-qc]

53. S.D. Odintsov, D. SÃąez-GÃşmez. Phys. Lett. B 725, 437 (2013). arXiv:1304.5411 [gr-qc]

54. D.A. Demir, C.N. Karahan, work in progress (2014) 